\title{
Prompt atmospheric neutrinos and muons: dependence on the gluon distribution function
}

\author{
Graciela Gelmini, ${ }^{1}$ Paolo Gondolo, ${ }^{2}$ Gabriele Varieschi ${ }^{1}$ \\ ${ }^{1}$ Dept. of Physics and Astronomy, UCLA (University of California, Los Angeles) \\ 405 Hilgard Ave., Los Angeles CA 90095, USA \\ gelmini,variesch@physics.ucla.edu \\ ${ }^{2}$ Max-Planck-Institut für Physik (Werner-Heisenberg-Institut) \\ Föhringer Ring 6, 80805 München, Germany \\ gondolo@mppmu.mpg.de
}

\begin{abstract}
We compute the next-to-leading order QCD predictions for the vertical flux of atmospheric muons and neutrinos from decays of charmed particles, for different PDF's (MRS-R1, MRS-R2, CTEQ$4 \mathrm{M}$ and MRST) and different extrapolations of these at small partonic momentum fraction $x$. We find that the predicted fluxes vary up to almost two orders of magnitude at the largest energies studied, depending on the chosen extrapolation of the PDF's. We show that the spectral index of the atmospheric leptonic fluxes depends linearly on the slope of the gluon distribution function at very small $x$. This suggests the possibility of obtaining some bounds on this slope in "neutrino telescopes", at values of $x$ not reachable at colliders, provided the spectral index of atmospheric leptonic fluxes could be determined.
\end{abstract}

\section{INTRODUCTION}

The flux of atmospheric neutrinos and muons at very high energies, above $1 \mathrm{TeV}$, originates primarily from semileptonic decays of charmed particles instead of pions and kaons, which are the dominant decay modes at lower energies (see for example [1]). This flux is one of the most important backgrounds for "neutrino telescopes", limiting their sensitivity to astrophysical signals, especially for future $\mathrm{km}^{3}$ detectors which might be able to observe neutrinos and muons at extremely high energies, even up to $10^{12} \mathrm{GeV}$.

We use perturbative QCD (pQCD), the theoretically preferred model, to compute the charm production. We perform a true next-to-leading order (NLO) pQCD analysis of the production of charmed particles in the atmosphere, together with a full simulation of the particle cascades down to the final muons and neutrinos. This is done by combining the NLO pQCD calculations of charm production and computer routines of Mangano, Nason, and Ridolfi [2.3] (called MNR in the following) with the computer simulations of the cascades generated by PYTHIA [4]. These are the same programs currently used to compare pQCD predictions with experimental data in accelerator experiments.

We have already presented results of our calculations in a previous paper [5] (called GGV1 from now on), in which all the details of the program we use can be found. The main goal of our first paper was to compare the fluxes obtained with the NLO and the leading order (LO) calculations, i.e. we computed the $K$ factor for the neutrino and muon fluxes. This was done to improve on the first study of atmospheric fluxes based on pQCD, performed by Thunman, Ingelman, and Gondolo a few years ago in Ref. [6] (called TIG in the following). TIG used the LO charm production cross section computed by PYTHIA, multiplied by a constant $K$ factor of 2 to bring it in line with the NLO values, and supplemented by parton shower evolution and hadronization according to the Lund model.

In GGV1 we found the $K$ factors for different parton distribution functions (PDF's), as function of energy, to be in a range between 2.1 and 2.5. A similar analysis was recently made in Pasquali, Reno, and Sarcevic [7] (called PRS from now on), with results compatible with ours, using a treatment of the problem complementary to ours. In fact, PRS used approximate analytic solutions to the cascade equations in the atmosphere, also introduced by TIG, while we make instead a full simulation of the cascades.

In GGV1 we showed that the approach used by TIG (i.e. multiplying the LO fluxes by an overall $K$ factor of 2 ) was essentially correct, except for their relative low $K$ factor (since $K$ values of $2.2-2.4$, depending slightly on the PDF, provide estimates of the NLO within about 10\%). However, while TIG found neutrino and muon fluxes lower than the lowest previous estimate, we found instead larger fluxes (by factors of 3 to 10 at the highest energies, about $10^{9} \mathrm{GeV}$ ), in the bulk part of previous predictions. The main reason for this difference is studied in this paper. 
Here we explore the dependence of the atmospheric fluxes on the extrapolation of the gluon PDF at very small partonic momentum fraction $x, x \lesssim 10^{-5}$, which is crucial for the fluxes at high energies. As explained below, the relevant momentum fraction $x$ of the interacting atmospheric parton is of the order of the inverse of the leptonic energy $E_{l}$ (in the atmospheric rest frame) in GeV. This energy, in turn, is of the order of $0.1 E$, where $E$ is the energy per nucleon of the incoming cosmic ray in the lab. frame (the atmospheric rest frame). Thus, for $E_{l} \gtrsim 10^{5} \mathrm{GeV}$, we need the PDF's at $x \lesssim 10^{-5}$, values of $x$ which are not reached experimentally. The final fluxes depend mostly on the gluon PDF, since this is by far the dominant one at these small $x$ values and charm is mostly produced through gluon-gluon fusion processes.

A concern that has been expressed to us several times is the applicability of the MNR NLO-pQCD calculations, mostly done for accelerator physics, to the different kinematic domain of cosmic rays. In response we remark that, for the less steep extrapolations of the gluon structure function $g(x)$ that we use at small $x$, we have large logarithms, known as " $\ln (1 / \mathrm{x})$ " terms, where $x \simeq \sqrt{4 m_{c}^{2} / s}, s$ is the hadronic center of mass squared energy and this $x$ is the average value of the hadron energy fraction needed to produce the $c \bar{c}$ pair. With the extrapolation $g(x) \simeq x^{\lambda-1}$ (see below) and $\lambda$ close to 0.5 , and possibly for the intermediate choices of $\lambda$ also, there should be no large logarithm. The problem arises for $\lambda$ too close to zero. We will attempt to deal with this problem in future work. Moreover, contrary to the case in accelerators, we do not have the uncertainty present in the differential cross sections [3] when $k_{T}$ is much larger than $m_{c}$, due to the presence of large logarithms of $\left(k_{T}^{2}+m_{c}^{2}\right) / m_{c}^{2}$. Because we do not have here a forward cut in acceptance, the characteristic transverse charm momentum in our simulations is of the order of the charm mass, $k_{T} \simeq O\left(m_{c}\right)$.

In this paper, as in GGV1, the MNR program is used to compute the inclusive charm cross section and the cascades simulated by PYTHIA are initiated by a single $c$ quark. This is the 'single' mode described in our previous paper GGV1, where we argued its advantages. We explained there our normalization of the NLO charm production cross sections in the MNR program, and described in detail the computer simulations used to calculate the neutrino and muon fluxes, which we briefly review in Sections II and III. Except for the inclusion of the NLO calculations our model closely follows TIG. In Section IV we show the neutrino and muon fluxes we obtain for different low $x$ behaviors of the gluon PDF and we compare them with the TIG fluxes. In Section $\mathrm{V}$, we give analytic arguments that explain and support our results.

Finally, as in GGV1 (and TIG), we consider only vertical showers for simplicity. We intend to study those from all directions in the future.

\section{CHARM PRODUCTION IN PQCD AND CHOICE OF PDF'S}

Our NLO calculation is based on the MNR computer code. The NLO cross section for charm production depends on the choice of the parton distribution functions and on three parameters: the charm quark mass $m_{c}$, the renormalization scale $\mu_{R}$, and the factorization scale $\mu_{F}$. In order to calibrate the charm production routines we fit the most recent experimental data 811 (differential and total cross sections) with one and the same combination of $m_{c}, \mu_{R}$, and $\mu_{F}$, for each PDF we use (see [5] for complete details). Several choices of $m_{c}, \mu_{R}$ and $\mu_{F}$ may work equally well. In fact the cross sections increase by decreasing $\mu_{F}, \mu_{R}$ or $m_{c}$, so changes in the three variables can be played against each other to obtain practically the same results. We use just one such choice for each PDF. We intend to further study the uncertainty related to this range of possible choices in the future.

As in GGV1, here we use the PDF's MRS R1, R2 [12] and CTEQ 4M [13], with the following parameters. We choose $\mu_{R}=m_{T}, \mu_{F}=2 m_{T}$ for all sets, where $m_{T}$ is the transverse mass, $m_{T}=\sqrt{k_{T}^{2}+m_{c}^{2}}$, and

$$
\begin{array}{ll}
m_{c}=1.185 \mathrm{GeV} & \text { for MRS R1, } \\
m_{c}=1.31 \mathrm{GeV} & \text { for MRS R2, } \\
m_{c}=1.27 \mathrm{GeV} & \text { for CTEQ 4M. }
\end{array}
$$

The data we use for this 'calibration' of the MNR program are shown in Table 1 and Table 2 of GGV1. In this paper, we add to our list of PDF's the latest of the MRS set, the MRST [14], with charm mass

$$
m_{c}=1.25 \mathrm{GeV} \text { for MRST, }
$$

obtained with the same procedure used for the other PDF's.

As we will see clearly in Sect. V, due to the steep decrease with increasing energy of the incoming flux of cosmic rays, only the most energetic charm quarks produced count, and these come from the interactions of projectile partons carrying a large fraction of the incoming nucleon momentum. Thus, the characteristic $x$ of the projectile parton, that 
we call $x_{1}$, is large. It is $x_{1} \simeq O\left(10^{-1}\right)$. We can, then, immediately understand that very small partonic momentum fractions are needed in our calculation, because typical partonic center of mass energies $\sqrt{\hat{s}}$ are close to the $c \bar{c}$ threshold, $2 m_{c} \simeq 2 \mathrm{GeV}$ (since the differential cross section decreases with increasing $\hat{s}$ ) while the total center of mass energy squared is $s=2 m_{N} E$ (with $m_{N}$ the nucleon mass, $m_{N} \simeq 1 \mathrm{GeV}$ ). Calling $x_{2}$ the momentum fraction of the target parton (in a nucleus of the atmosphere), then $x_{1} x_{2} \equiv \hat{s} / s=4 m_{c}^{2} /\left(2 m_{N} E\right) \simeq \mathrm{GeV} / E$. Thus, $x_{2} \simeq O(\mathrm{GeV} / 0.1 E)$, where $E$ is the energy per nucleon of the incoming cosmic ray in the lab. frame. The characteristic energy $E_{c}$ of the charm quark and the dominant leptonic energy $E_{l}$ in the fluxes are $E_{l} \simeq E_{c} \simeq 0.1 E$, thus $x_{2} \simeq O\left(\mathrm{GeV} / E_{l}\right)$. Namely $x_{2} \simeq 10^{-6}, 10^{-7}$ at $E_{l} \simeq 1,10 \mathrm{PeV}$.

For $x>10^{-5}\left(E \lesssim 10^{3} \mathrm{TeV}\right)$, PDF's are available from global analyses of existing data. We use four sets of PDF's. Three of these, MRS R1, MRS R2 [12] and CTEQ 4M [13] (used also in GGV1), incorporate most of the latest HERA data and cover the range of parton momentum fractions $x \geq 10^{-5}$ and momentum transfers $Q^{2} \geq 1.25-2.56 \mathrm{GeV}^{2}$. MRS R1 and MRS R2 differ only in the value of the strong coupling constant $\alpha_{s}$ at the Z boson mass: in MRS R1 $\alpha_{s}\left(M_{Z}^{2}\right)=0.113$, and in MRS R2 $\alpha_{s}\left(M_{Z}^{2}\right)=0.120$. The former value is suggested by "deep inelastic scattering" experiments, and the latter by LEP measurements. This difference leads to different values of the PDF parameters at the reference momentum $Q_{0}^{2}=1.25 \mathrm{GeV}^{2}$, where the QCD evolution of the MRS R1 and R2 PDF's is started. The CTEQ 4M is the standard choice in the $\overline{M S}$ scheme in the most recent group of PDF's from the CTEQ group $\left(\alpha_{s}\left(M_{Z}^{2}\right)=0.116\right.$ for CTEQ 4M). In this paper we also use the very recent MRST [14]. This new PDF set includes all the latest experimental measurements that have become available and, for the first time, an investigation of the uncertainty in the gluon distribution function. We will use the main choice of the MRST set, the "central gluon" MRST, the central value of the gluon PDF's of the package, which is considered the optimum global choice of this new set. The range in $Q^{2}$ and $x$ of MRST set is the same as for the older MRS R1-R2 $\left(x \geq 10^{-5}\right.$ and $\left.Q^{2} \geq 1.25 \mathrm{GeV}^{2}\right)$, and $\alpha_{s}\left(M_{Z}^{2}\right)=0.1175$.

For $x \ll 1$, all these PDF's go as

$$
x f_{i}\left(x, Q^{2}\right) \simeq A_{i} x^{-\lambda_{i}\left(Q^{2}\right)},
$$

where $i$ denotes valence quarks $u_{v}, d_{v}$, sea quarks $S$, or gluons $g$. The PDF's we used have $\lambda_{S}\left(Q_{0}^{2}\right) \neq \lambda_{g}\left(Q_{0}^{2}\right)$, in contrast to older sets of PDF's which assumed an equality. As $x$ decreases the density of gluons grows rapidly. At $x \simeq 0.3$ it is comparable to the quark densities but, as $x$ decreases it increasingly dominates over them. Quark densities become negligible at $x \lesssim 10^{-3}$.

The PDF's need to be extrapolated to $x<10^{-5}\left(E \gtrsim 10^{3} \mathrm{TeV}\right)$. Extrapolations based on Regge analysis usually propose $x g(x) \sim x^{-\lambda}$ with $\lambda \simeq 0.08$ [15, while evolution equations used to resum the large logarithms $\alpha_{s} \ln (1 / x)$ mentioned before, such as the BFKL (Balitsky, Fadin, Kuraev, Lipatov [16]) find also $x g(x) \sim x^{-\lambda}$, but with $\lambda \simeq 0.5$.

In this work we use extrapolations with different values of $\lambda$. For the older MRS R1-R2 and CTEQ 4M we consider only the two extreme behaviors and the intermediate one that we used in GGV1, namely: (i) a constant extrapolation $\lambda_{g}\left(Q^{2}\right)=0$ for $x \leq 10^{-5}$; (ii) a linear extrapolation of $\ln g(x)$ as a function of $\ln x, \ln g(x)=-\left(\lambda_{g}\left(Q^{2}\right)+1\right) \ln x+\ln A_{g}$, where $\lambda_{g}\left(Q^{2}\right)$ is taken at $x=10^{-5}$, the smallest $x$ for which the PDF's are provided (we call $\lambda(\mathrm{R} 1), \lambda(\mathrm{R} 2)$ or $\lambda(4 \mathrm{M})$ the $\lambda$ 's so obtained); (iii) an extrapolation with $\lambda_{g}\left(Q^{2}\right)=0.5$ for $x \leq 10^{-5}$. Cases (i) and (iii) are extreme choices theoretically justified before [15], while (ii) is somewhat in between, with a resulting $\lambda \simeq 0.2-0.3$.

For the new MRST we have included several values of $\lambda$, in order to test the dependence on this parameter in a more complete way: (i) extrapolations with different $\lambda$ 's, i.e. $\lambda_{g}\left(Q^{2}\right)=0,0.1,0.2,0.3,0.4,0.5$ for $x \leq 10^{-5}$; (ii) we also included the linear extrapolation of $\ln g(x)$ as a function of $\ln x$, similar to the second intermediate choice of the previous list; we will call $\lambda(\mathrm{T})$ the $\lambda$ obtained in this way.

\section{SIMULATION OF PARTICLE CASCADES IN THE ATMOSPHERE}

In this section we briefly describe the computer simulation used to calculate the neutrinos and muons fluxes; a more detailed description can be found in GGV1 [5]. The charm production process in the atmosphere and the particle cascades are simulated by modifying and combining together two different programs: the MNR routines [3] and PYTHIA 6.115 [4].

The MNR program was modified to become an event generator for charm production at different heights in the atmosphere and for different energies of the incoming primary cosmic rays.

The charm quarks (and antiquarks) generated by this first stage of the program are then fed into a second part which handles quark showering, fragmentation and the interactions and decays of the particles down to the final leptons. The cascade evolution is therefore followed throughout the atmosphere: the muon and neutrino fluxes at sea level are the final output of the process. 
In order to make our results comparable to those of TIG, we keep the same modeling of the atmosphere and of the primary cosmic ray flux as in TIG and the same treatment of particle interactions and decays in the cascade.

We recall however that our main improvements are the inclusion of a true NLO contribution for charm production, the use of updated PDF's and, in this second paper, the different extrapolations used for the gluon PDF at low $x$.

In the rest of this section we review briefly the model for the atmosphere and the primary flux used in this study, which is the same of GGV1 and was introduced originally by TIG.

We assume a simple isothermal model for the atmosphere. Its density at vertical height $h$ is

$$
\rho(h)=\frac{X_{0}}{h_{0}} e^{-h / h_{0}},
$$

with the parameters, scale height $h_{0}=6.4 \mathrm{~km}$ and column density $X_{0}=1300 \mathrm{~g} / \mathrm{cm}^{2}$ at $h=0$, chosen as in TIG to fit the actual density in the range $3 \mathrm{~km}<h<40 \mathrm{~km}$, important for cosmic ray interactions. Along the vertical direction, the amount of atmosphere traversed by a particle, the depth $X$, is related to the height $h$ simply by

$$
X=\int_{h}^{\infty} \rho\left(h^{\prime}\right) d h^{\prime}=X_{0} e^{-h / h_{0}} .
$$

The atmospheric composition at the important heights is approximately constant: $78.4 \%$ nitrogen, $21.1 \%$ oxygen and $0.5 \%$ argon with average atomic number $\langle A\rangle=14.5$.

Following TIG [6], we neglect the detailed cosmic ray composition and consider all primaries to be nucleons with energy spectrum

$$
\begin{aligned}
\phi_{N}(E, 0)\left[\frac{\text { nucleons }}{\mathrm{cm}^{2} \mathrm{~s} \mathrm{sr} \mathrm{GeV} / \mathrm{A}}\right]=\phi_{0} E^{-\gamma-1}= \\
\qquad= \begin{cases}1.7(E / \mathrm{GeV})^{-2.7} & \text { for } E<510^{6} \mathrm{GeV} \\
174(E / \mathrm{GeV})^{-3} & \text { for } E>510^{6} \mathrm{GeV}\end{cases}
\end{aligned}
$$

The primary flux is attenuated as it penetrates into the atmosphere by collisions against the air nuclei. An approximate expression for the intensity of the primary flux at a depth $X$ is (see [6] again)

$$
\phi_{N}(E, X)=e^{-X / \Lambda_{N}} \phi_{N}(E, 0) .
$$

The nuclear attenuation length $\Lambda_{N}$, defined as

$$
\Lambda_{N}(E)=\frac{\lambda_{N}(E)}{1-Z_{N N}(E)},
$$

has a mild energy dependence through $\lambda_{N}$ and $Z_{N N}$, the spectrum-weighted moment for nucleon regeneration in nucleon-nucleon collisions. We use the $Z_{N N}$ values in Fig. 4 of Ref. [6]. The interaction thickness $\lambda_{N}$ is

$$
\lambda_{N}(E, h)=\frac{\rho(h)}{\sum_{A} \sigma_{N A}(E) n_{A}(h)},
$$

where $n_{A}(h)$ is the number density of air nuclei of atomic weight $A$ at height $h$ and $\sigma_{N A}(E)$ is the total inelastic cross section for collisions of a nucleon $N$ with a nucleus $A$. This cross section scales essentially as $A^{2 / 3}, \sigma_{N A}(E)=$ $A^{2 / 3} \sigma_{N N}(E)$. For $\sigma_{N N}(E)$ we use the fit to the available data in Ref. [17]. Using our height independent atmospheric composition, we simplify Eq. (11) as follows,

$$
\lambda_{N}(E, h)=\frac{\langle A\rangle}{\left\langle A^{2 / 3}\right\rangle} \frac{\mathrm{u}}{\sigma_{N N}(E)}=2.44 \frac{\mathrm{u}}{\sigma_{N N}(E)} .
$$

Here \langle\rangle denotes average and $\mathrm{u}$ is the atomic mass unit, that we write as

$$
\mathrm{u}=1660.54 \mathrm{mb} \mathrm{g} / \mathrm{cm}^{2} .
$$

Therefore in our approximations $\lambda_{N}(E)$ is independent of height. 


\section{NEUTRINO AND MUON FLUXES}

We present here the results of our simulations with all the PDF's and the values of $\lambda$ described in Section I1].

The NLO total inclusive charm-anticharm production cross sections $\sigma_{c \bar{c}}$ for our four different PDF's are shown in Fig. 1 over the energy range needed by our program, $E \leq 10^{11} \mathrm{GeV}$. In the top part of the figure we compare the results of MRS R1-R2 and CTEQ 4M (with their different values of $\lambda$ described before) to the cross section used in the TIG model. In the bottom part we show the same comparison, done just with the new MRST, with its different $\lambda$ 's (in all these figures cross sections increase for increasing values of $\lambda$ ).

All these cross sections were calculated using the MNR program, with the 'calibration' described in Sect. III, up to the NLO contribution. We can see in the figure that all our cross sections agree at low energies, as expected due to our 'calibration' at $250 \mathrm{GeV}$, and are very similar for energies up to $10^{6} \mathrm{GeV}$. Beyond this energy they start showing their dependence on the $\lambda$ value and also a slight dependence on the PDF used, which was already noticed in GGV1. As it can be seen from both parts of the figure, the increase of the cross sections with $\lambda$ is evident at the highest energies: at the maximum energy considered the cross sections for the two extreme values of $\lambda$ differ by almost a factor of ten.

We also notice that, for energies above $10^{4} \mathrm{GeV}$, our cross sections are always considerably higher than the one used by TIG. As we have already explained in GGV1, TIG used an option of PYTHIA by which the gluon PDF is extrapolated for $x \leq 10^{-4}$ with $\lambda=0.08$. In fact the TIG cross section at the highest energies shows the same slope of our results for $\lambda \simeq 0$, but is always lower than our lowest cross sections by about a factor of three.

This can be explained only in part by the fact that the TIG cross section up to NLO is the LO result obtained with PYTHIA, multiplied by a constant $K$ factor of 2, while at large energies the $K$ factor (see GGV1 for details) is actually larger than 2 by about 10-15\%. The bulk of the difference is however due to the different evaluations of the cross sections, even at LO, done by the MNR routines (our method) and directly by PYTHIA (approach used by TIG).

Our results for the prompt fluxes are shown in Figs. 2-5, for MRS R1-R2, CTEQ 4M and MRST.

In Figs. 2 and 3 we show the $E_{\ell}^{3}$-weighted vertical prompt fluxes $E_{\ell}^{3} \phi_{\ell}$, calculated to NLO, for muons and muonneutrinos, together with the fluxes from TIG, both from prompt and conventional sources (dotted lines). The flux of electron-neutrinos is practically the same as that of muon-neutrinos. Fig. 4 describes the spectral index of the differential fluxes, defined as $\alpha_{\ell}=-\partial \ln \phi_{\ell} / \partial \ln E_{\ell}$.

The effects of the different extrapolations of $g(x)$ to $x<10^{-5}$ (see Sect. III) are noticeable at $E_{\ell} \gtrsim 10^{5} \mathrm{GeV}$. In Figs. 2 and 3, the $E_{\ell}^{3}$-weighted fluxes increase with $\lambda$ : they can differ by up to two orders of magnitude at the highest energy considered, $10^{9} \mathrm{GeV}$, for the two extreme choices of $\lambda$. This behavior is similar for all the PDF's considered.

The $\lambda$ dependence of the fluxes can also affect the energy at which the prompt contribution dominates over the conventional sources: this is particularly true for the muon fluxes as it can be seen in Fig. 2; for the $\nu_{\mu}+\bar{\nu}_{\mu}$ fluxes this effect is less important (see Fig. 3) and it doesn't exist for the $\nu_{e}+\overline{\nu_{e}}$ fluxes, for which the conventional contribution is much lower. Apart from these differences due to the $\lambda$ values, charm decay dominates over conventional sources at $E_{\mu} \gtrsim 10^{6} \mathrm{GeV}$ for muons, $E_{\nu_{\mu}} \gtrsim 10^{5} \mathrm{GeV}$ for muon-neutrinos, and $E_{\nu_{e}} \gtrsim 10^{4} \mathrm{GeV}$ for electron-neutrinos.

We also see that all our fluxes for $\lambda \simeq 0$ are similar to those of TIG at energies above $10^{6} \mathrm{GeV}$. We have already mentioned that TIG used a very low value of $\lambda, \lambda=0.08$. It is remarkable that, for these low values of $\lambda$, we obtain similar final fluxes in spite of the differences of the two simulations and of the total cross sections already noted in Fig. 1.

We can also compare our fluxes to those of the recent PRS results |7]. As we have already noticed in GGV1, for intermediate values of $\lambda$ our results are very similar to the PRS ones. From Fig. 3, for example, we see that our fluxes for the $\lambda=0.3$ case (calculated with MRST) are close to the corresponding PRS results shown in Fig. 8 of Ref. [7], calculated with CTEQ 3M and $\lambda \simeq 0.3$. Our results are lower than the PRS by $30-50 \%$ at the highest energies, which is probably due to the PDF's used and to the different approach of the two groups.

Regarding the dependence of the spectral index $\alpha_{\ell}$ on the slope $\lambda$ of the gluon PDF, we notice in Fig. 4 that, for all four PDF's, above about $10^{6} \mathrm{GeV}$ the differences in slope between the $\lambda=0$ and $\lambda=0.5$ fluxes is about 0.5 , suggesting that the spectral index is $\alpha_{\ell}\left(E_{\ell}\right)=b_{\ell}\left(E_{\ell}\right)-\lambda$, namely,

$$
\phi_{\ell}\left(E_{\ell}\right) \sim E_{\ell}^{-\alpha_{\ell}\left(E_{\ell}\right)}=E_{\ell}^{-b_{\ell}\left(E_{\ell}\right)+\lambda},
$$

where $b_{\ell}\left(E_{\ell}\right)$ is an energy dependent coefficient, that can be read off directly from the $\lambda=0$ curve $\left(b_{\ell}\left(E_{\ell}\right)\right.$ is the spectral index for $\lambda=0$ ). We will justify this result in Sect. $\mathrm{V}$. Due to this linear dependence of the spectral index on $\lambda$, given a model which specifies the function $b_{\ell}\left(E_{\ell}\right)$, the value of $\lambda$ could be determined through a measurement of any of the $\phi_{\ell}$ fluxes at two different energies. We will study in detail this possibility elsewhere [18].

Here we only comment on the typical rates in a $\mathrm{km}^{3}$ detector. It can be estimated from the curves of Fig. 2 that the number of prompt atmospheric muons traversing a $\mathrm{km}^{3}$ detector from above would be over 100 per year around 
a muon energy of $1 \mathrm{PeV}$, decreasing rapidly to less than 1 per year above $100 \mathrm{PeV}$. In this energy range there is a concrete possibility of detecting these prompt muons. Notice that the intensity of the prompt muon flux depends critically on the value of $\lambda$, suggesting still another way to estimate $\lambda$ through the measurement of the fluxes.

In Fig. 5 we study the dependence of the prompt fluxes on the PDF for fixed values of $\lambda$. We summarize our previous results for $\lambda=0$ (left) and for $\lambda=0.5$ (right), and compare them again to TIG. The figures on the top show the $E_{\ell}^{3}$-weighted fluxes, those on the bottom the spectral indices. As we already noticed in GGV1, the dependence on the PDF is not strong, all fluxes are very similar. This indicates that our procedure for the 'calibration' of our simulation with different PDF's (described in Sect. II) is good. There are, however, some differences between the PDF's: in some cases (especially for $\lambda=0$ ) the results of MRS R2 and CTEQ 4M are very similar and higher than those of MRS R1 and MRST (also very close to each other). The maximum difference between all these fluxes is at the level of 30 to $70 \%$ at high energies.

We want here to remark once more that our $\lambda=0$ fluxes are very close to that of TIG at energies above $10^{6}$ GeV (and also below $10^{3} \mathrm{GeV}$, but the prompt fluxes are not important at these low energies). For increasing values of $\lambda$, our results are higher than TIG, even by two orders of magnitude for $\lambda=0.5$ and at the highest energies. From the bottom part of the figure we notice that also the spectral indices are almost independent of the PDF used. This indicates that the linear dependence between $\alpha_{\ell}$ and $\lambda$ of Eq. (14) is not affected by the choice of the PDF and again might be used to determine the value of $\lambda$. We will return on this analysis in more details in another paper 18 .

\section{ANALYTIC INSIGHT}

In this section we first find the characteristic values of the partonic momentum fractions in the cosmic ray nucleus and in the nucleus in the atmosphere, and then derive the linear relation between the slope of the atmospheric muon (or neutrino) fluxes and the slope of the gluon parton distribution function.

We first show that the characteristic values of the partonic momentum fractions of the incoming cosmic ray parton, $x_{1}$, and of the target parton belonging to a nucleus in the atmosphere, $x_{2}$, are respectively,

$$
x_{1} \simeq 10^{-1} \quad x_{2} \simeq(E / 10 \mathrm{GeV})^{-1}
$$

where $E$ is the energy of the incoming nucleon (a proton in this paper) in the atmosphere reference frame. Precisely because of the small value of $x_{2}$, for the relevant energies $E \gtrsim 10^{4} \mathrm{GeV}$ the gluon density $g\left(x_{2}\right)$ is much larger than the density of quarks, which we, thus, neglect in these analytic arguments.

Let us first consider the charm flux at production $d \phi_{c}\left(E_{c}, X\right) / d X$, defined as the rate of c quark production? per unit area, unit depth and unit charm energy $\left(E_{c}\right.$ in the atmosphere reference frame) in the interactions of the attenuated nucleon flux $\phi_{N}(E, X)$ with the air nuclei in the atmospheric layer between $X$ and $X+d X$. To obtain $d \phi_{c}\left(E_{c}, X\right) / d X$ for a layer of transverse area $\mathcal{A}$ and height $|d h|$, we simply multiply the $c$ production rate per air nucleus (which equals the incoming nucleon flux at depth $X$ times the cross section for $N+A \rightarrow c+Y$, where $Y$ stands for "anything" and $\mathrm{N}$ is simply a proton $\mathrm{p}$ in our study) by the number of nuclei $A$ in the layer (which is $\left.\mathcal{A}|d h| n_{A}(h)\right)$ and divide the result by the transverse area $\mathcal{A}$ and the layer thickness $d X=\rho(h)|d h|$. We find

$$
\frac{d \phi_{c}\left(E_{\mathrm{c}}, X\right)}{d X}=\sum_{\mathrm{A}} \frac{n_{\mathrm{A}}(h)}{\rho(h)} \int_{E_{\mathrm{c}}}^{\infty} d E \phi_{\mathrm{N}}(E, X) \frac{d \sigma\left(\mathrm{pA} \rightarrow \mathrm{cY} ; \mathrm{E}, \mathrm{E}_{\mathrm{c}}\right)}{d E_{\mathrm{c}}}
$$

We assume that the charm production cross section simply scales as $A$, which is expected when it is much smaller than the total inelastic cross section. In this case, the sum over $A$ becomes trivial, and we have ( $u$ is the atomic mass unit)

$$
\frac{d \phi_{c}\left(E_{\mathrm{c}}, X\right)}{d X}=\frac{1}{\mathrm{u}} \int_{E_{c}}^{\infty} d E \phi_{N}(E, X) \frac{d \sigma\left(p N \rightarrow c Y ; E, E_{c}\right)}{d E_{c}}
$$

\footnotetext{
${ }^{1}$ This is what we compute in our simulations (we use our 'single' mode), only the production of a c quark is calculated. Then the result is multiplied by two to include the contribution of the antiquark (see [5] for details).
} 
In these analytical considerations, we assume a simple power law for the primary flux and an energy independent attenuation length.2 With these approximations, the attenuated primary flux reads (see Eqs. 8-13)

$$
\phi_{N}(E, X)=\phi(X) E^{-\gamma-1},
$$

where $\phi(X)=\phi_{0} \exp \left(-X / \Lambda_{N}\right)$. Substituting this approximate expression for the attenuated primary flux and changing the integration variable from $E$ to $x_{E}=E_{c} / E$ in Eq. (17), we find

$$
\frac{d \phi_{c}\left(E_{\mathrm{c}}, X\right)}{d X}=\frac{\phi(X)}{\mathrm{u}} E_{c}^{-\gamma-1} \int_{0}^{1} d x_{E} x_{E}^{\gamma} \frac{d \sigma\left(p N \rightarrow c Y ; x_{E}, E_{c}\right)}{d x_{E}} .
$$

The differential cross section $d \sigma(p N \rightarrow c Y) / d x_{E}$ is given in terms of the partonic differential cross section $d \hat{\sigma}_{i j} / d x_{E}$ (where $i$ and $j$ are partons belonging to the projectile 1 and the target 2 respectively), and the PDF's $f_{i}^{1}\left(x_{1}, \mu_{F}^{2}\right)$ and $f_{j}^{2}\left(x_{2}, \mu_{F}^{2}\right)$ as

$$
\frac{d \sigma(p N \rightarrow c Y)}{d x_{E}}=\sum_{i j} \int d x_{1} d x_{2} f_{i}^{1}\left(x_{1}, \mu_{F}^{2}\right) f_{j}^{2}\left(x_{2}, \mu_{F}^{2}\right) \frac{d \hat{\sigma}_{i j}}{d x_{E}}
$$

Here $x_{1}$ and $x_{2}$ are the momentum fractions of the projectile and target partons. Mangano et al. [3] give the partonic cross section in terms of functions $h_{i j}$ as

$$
E_{c} \frac{d \hat{\sigma}_{i j}}{d^{3} k}=\frac{\alpha_{s}^{2}\left(\mu_{R}\right)}{\hat{s}^{2}} h_{i j}\left(\tau_{x}, \tau_{2}, \rho, \mu_{R}, \mu_{F}\right)
$$

where $k$ and $E_{c}$ are the momentum and energy of the produced $c$ quark, and, in the notation of Ref. [3], $\rho \equiv 4 m_{c}^{2} / \hat{s}$, $\tau_{x}=1-\tau_{1}-\tau_{2}, \tau_{1} \equiv\left(k \cdot p_{1} / p_{1} \cdot p_{2}\right), \tau_{2} \equiv\left(k \cdot p_{2} / p_{1} \cdot p_{2}\right)$ and $\hat{s} \equiv\left(p_{1}+p_{2}\right)^{2}$, while $p_{1}$ and $p_{2}$ are the projectile and target parton momenta respectively, $p_{1}=x_{1} P_{1}, p_{2}=x_{2} P_{2}$. The hats indicate quantities in the partonic center of mass (those without hats are in the lab. frame at rest with the atmosphere).

In the partonic center of mass frame, the projectile and target parton momenta are

$$
\hat{p_{1}}=\left(\frac{\sqrt{\hat{s}}}{2}, 0,0, \frac{\sqrt{\hat{s}}}{2}\right), \hat{p_{2}}=\left(\frac{\sqrt{\hat{s}}}{2}, 0,0,-\frac{\sqrt{\hat{s}}}{2}\right), \hat{k}=\left(\hat{E}_{c}, 0, \hat{k}_{T}, \hat{k}\right),
$$

and we have

$$
\tau_{2}=\frac{\hat{E}_{c}+\hat{k}}{\sqrt{\hat{s}}}, \quad \tau_{x}=1-\frac{2 \hat{E}_{c}}{\sqrt{\hat{s}}} .
$$

Then, after integration over azimuthal angles,

$$
\frac{d^{3} k}{E_{c}}=\frac{d^{3} \hat{k}}{\hat{E}_{c}}=2 \pi d \hat{E}_{c} d \hat{k}=\pi \hat{s} d \tau_{2} d \tau_{x}
$$

The kinematic bounds $m_{c} \leq \hat{E}_{c} \leq \sqrt{\hat{s}} / 2$ and $|\hat{k}| \leq \sqrt{\hat{E}_{c}^{2}-m_{c}^{2}}$ fix the integration domains of $\tau_{2}$ and $\tau_{x}$. Using $\rho=4 m_{c}^{2} / \hat{s}$, we get $(1-\sqrt{1-\rho}) / 2 \leq \tau_{2} \leq(1+\sqrt{1-\rho}) / 2$ and $0 \leq \tau_{x} \leq 1-\tau_{2}-\left(\rho / 4 \tau_{2}\right)$. We can use the relation

$$
x_{E}=\frac{E_{c}}{E}=\frac{k \cdot P_{2}}{P_{1} \cdot P_{2}}=x_{1} \frac{k \cdot p_{2}}{p_{1} \cdot p_{2}}=x_{1} \tau_{2}
$$

to write the differential cross section in $d x_{E}$ as

\footnotetext{
2 The dependence of $\Lambda_{N}$ on $E$ is actually very mild. In fact the whole factor $e^{-X / \Lambda_{N}(E)}$ behaves like $E^{-\beta}$ with $\beta \simeq 0.1$ for $E \gtrsim 10^{6} \mathrm{GeV}$ and $\beta$ even smaller for $E \lesssim 10^{6} \mathrm{GeV}$. Including this contribution in our analytic argument would just mean to replace $\gamma$ with $\gamma+\beta$ everywhere, i.e. the total spectral index would become $\gamma+1+\beta \simeq 3.1$ instead of 3.0, for energies above the knee at $E=510^{6} \mathrm{GeV}$. This slight change can actually be seen in our results of Fig. $7 \mathrm{~b}$ (see the description of that figure).
} 


$$
\frac{d \hat{\sigma}_{i j}}{d x_{E}}=\int d^{3} k \frac{d \hat{\sigma}_{i j}}{d^{3} k} \delta\left(x_{E}-x_{1} \tau_{2}\right)
$$

The bound $x_{1} x_{2}=\hat{s} / s \geq 4 m_{c}^{2} / 2 m_{p} E=4 \epsilon x_{E}$ ( $m_{p}$ is the proton mass, $\left.m_{p} \simeq 1 \mathrm{GeV}\right)$, where we define

$$
\epsilon=\frac{m_{c}^{2}}{2 m_{p} E_{c}}
$$

implies that $x_{1}$ and $x_{2}$ have a minimum lower bound larger than zero. In fact, $x_{1} \geq 4 \epsilon x_{E} / x_{2} \geq 4 \epsilon x_{E}$ (since $x_{2} \leq 1$ ). Taking $x_{1}$ as the independent variable, then $4 \epsilon x_{E} \leq x_{1} \leq 1$ and $4 \epsilon x_{E} / x_{1} \leq x_{2} \leq 1$. We now change the order of the integrations, in order to perform the integration in $x_{E}$ before the integrations in $x_{1}, x_{2}$ and $\tau_{2}$.

The integration over $x_{E}$ in Eq. (19) then becomes trivial, amounting to the replacement of $x_{E}^{\gamma}$ by $x_{1}^{\gamma} \tau_{2}^{\gamma}$, except for the necessary changes in the integration domains which become $0 \leq x_{1}, x_{2}, \tau_{2} \leq 1$ and $0 \leq x_{E} \leq\left(x_{1} x_{2} / \epsilon\right) \tau_{2}\left(1-\tau_{2}\right)$. For the $\delta\left(x_{E}-x_{1} \tau_{2}\right)$ in Eq. (26) to yield a non-zero result, we need to take $0 \leq x_{1} \tau_{2} \leq\left(x_{1} x_{2} / \epsilon\right) \tau_{2}\left(1-\tau_{2}\right)$, which means that $\tau_{2} \leq 1-\left(\epsilon / x_{2}\right)$, and given that $\tau_{2} \geq 0$, this means $x_{2} \geq \epsilon$. This leads to a factorization of the $x_{1}$ and $x_{2}$ integrations as follows:

$$
\begin{aligned}
& \int_{0}^{1} d x_{E} x_{E}^{\gamma} \frac{d \sigma(p N \rightarrow c Y)}{d x_{E}}=\frac{\pi \alpha_{s}^{2}\left(\mu_{R}\right)}{m_{c}^{2}} \times \\
& \sum_{i j}\left[\int_{0}^{1} d x_{1} x_{1}^{\gamma} f_{i}^{1}\left(x_{1}, \mu_{F}^{2}\right)\right]\left[\int_{\epsilon}^{1} d x_{2} f_{j}^{2}\left(x_{2}, \mu_{F}^{2}\right) \zeta_{i j}\left(\frac{\epsilon}{x_{2}}, \mu_{R}, \mu_{F}\right)\right],
\end{aligned}
$$

where the functions $\zeta_{i j}$ are defined as

$$
\zeta_{i j}\left(v, \mu_{R}, \mu_{F}\right)=v \int_{0}^{1-v} d \tau_{2} \tau_{2}^{\gamma+1} \int_{0}^{1-v-\tau_{2}} d \tau_{x} h_{i j}\left(\tau_{x}, \tau_{2}, 4 v \tau_{2}, \mu_{R}, \mu_{F}\right)
$$

and the argument $v$ is $v \equiv \epsilon / x_{2}$ (to rewrite the integration in $\tau_{2}$ we noticed that $\rho / 4 \tau_{2}=v$ ). The functions $h_{i j}$ are given by $h_{i j}\left(\tau_{x}, \tau_{2}, \rho, \mu_{R}, \mu_{F}\right)=h_{i j}^{(0)}\left(\tau_{2}, \rho\right) \delta\left(\tau_{x}\right)+O\left(\alpha_{s}^{2}\right)$. We will take only gluons as partons from now on, thus $f_{i}^{1}\left(x, \mu_{F}^{2}\right)=f_{j}^{2}\left(x, \mu_{F}^{2}\right)=g\left(x, \mu_{F}^{2}\right)$.

The function $\zeta_{g g}$, using $h_{g g}$ at the Born level, is shown in Fig. 6a for $\gamma=1.7$ and 2 (corresponding to the spectral indices $\gamma+1$ of the primary flux above and below the knee). In the same figure we see that the maximum of $\zeta_{g g}(v)$ is at $v \simeq 0.1$, namely $x_{2} \simeq 10 \epsilon$. However, given that $g\left(x_{2}, \mu_{F}^{2}\right)$ is a sharply increasing function with decreasing $x_{2}$ (i.e. for increasing $v$ at fixed $\left.E_{c}\right)$, the maximum of the product $g\left(x_{2}, \mu_{F}^{2}\right) \zeta_{g g}(v)$ is always to the right of the maximum of $\zeta_{g g}(v)$, at $v>0.1$. Therefore, the integral in $x_{2}$ in Eq. (28) is dominated by the values of $x_{2}$ of order $\epsilon$, namely

$$
x_{2} \simeq \epsilon \simeq \frac{\mathrm{GeV}}{2 E_{c}} .
$$

Returning to Eq. (28), the integral in $x_{1}$ shows that large values of $x_{1}$ will be dominant since $x_{1}^{\gamma} g\left(x_{1}\right) \rightarrow x_{1}^{\gamma-\lambda-1}$ for small $x$, where the exponent is positive, since $\gamma=1.7$ or 2 , while $0 \lesssim \lambda \lesssim 0.5$ (thus $\gamma-\lambda-1>0$ ). To see more precisely what range of $x_{1}$ dominates the integral, it is necessary to prove two statements. The first is that $\tau_{2} \equiv x_{E} / x_{1}<1$, due to kinematical constrains, therefore $x_{1}>x_{E}$. The second is that the characteristic value of $x_{E}$ is 0.1 , namely that the $c$-quark is mainly produced with 0.1 of the proton energy

$$
E_{c}=\mathcal{O}(0.1 E) \text {. }
$$

With respect to the kinematical limit on $\tau_{2}$, as we already mentioned, $\tau_{2} \equiv x_{E} / x_{1} \leq 1-v$, and we obtained as a kinematical constraint that $\epsilon \leq v=\epsilon / x_{2} \leq 1$ (since $x_{2}$ goes from $\epsilon$ to 1 ). Thus, $\tau_{2} \leq 1-\epsilon<1$, since $\epsilon$ is always larger than zero. Another way of obtaining this bound is the following. Since the partonic processes involved are $g g \rightarrow c \bar{c}$ or $g g \rightarrow c \bar{c} g$, then $\sqrt{\hat{s}} \geq 2\left(\hat{E}_{c}\right)_{\max }$ and due to $m_{c} \neq 0,(\hat{k})_{\max }<\left(\hat{E}_{c}\right)_{\max }$, therefore $\tau_{2}<2\left(\hat{E}_{c}\right)_{\max } / \sqrt{\hat{s}} \leq 1$.

That in fact $E_{c}=\mathcal{O}(0.1 E)$ is clearly demonstrated in Fig. 6b, which shows the function $x_{E}^{\gamma}\left(d \sigma / d x_{E}\right)$ normalized by the total c-production cross section. Thus we have proven that the dominant range of $x_{1}$ in Eq. (28) is $x_{1} \gtrsim \mathcal{O}(0.1 E)$ and also, combining together Eq. (30) and Eq. (31), our statement in Eq. (15) about $x_{2}$.

Even if we have not yet included gluon shadowing in our calculations, we want to point out that this effect might only be important for the target gluon (given that $x_{2}$ is very small) but it is not important for the gluons in the projectile (given that $x_{1} \gtrsim 0.1$ ). This means that the uncertainties on the composition of cosmic rays will not affect the results through shadowing effects. 
As a summary of our arguments we can say that, due to the incoming flux being rapidly falling with increasing energy of the primary, only the charm quarks produced with a large fraction of the incoming energy, $E_{c} \simeq 0.1 E$, count in the charm flux at production, and those highly energetic $c$ quarks come from projectile partons carrying a large fraction of the incoming momentum $x_{1} \gtrsim x_{E} \simeq 0.1$. On the other hand, because typical partonic center of mass energies $\sqrt{\hat{s}}$ are close to the $c \bar{c}$ threshold, $2 m_{c} \simeq 2 \mathrm{GeV}$ (since the cross section decreases steeply with increasing $\sqrt{\hat{s}}$ ), while the total center of mass energy squared is $s=2 m_{p} E$ (with $m_{p}$ the proton mass, $m_{p} \simeq 1 \mathrm{GeV}$ ), the product $x_{1} x_{2} \equiv \hat{s} / s=4 m_{c}^{2} /\left(2 m_{p} E\right) \simeq \mathrm{GeV} / E$. This shows that $x_{2} \simeq\left(\mathrm{GeV} / E x_{1}\right) \simeq \mathrm{GeV} / 0.1 E$.

We now derive the dependence on $\lambda$ of the muon and neutrino fluxes for a simple power law primary flux.

We can explain first the dependence on $\lambda$ of the spectral index of $d \phi_{c} / d X$ at large energies, and then, using this result, the dependence on $\lambda$ of the spectral indices of atmospheric muons and neutrinos. To start with, we notice that the integral in Eq. (28) depends on the charm energy $E_{c}$ only through the presence of the parameter $\epsilon$ in the integration on $x_{2}$. To approximately perform this integration at large energies, let us replace $g\left(x_{2}\right) \simeq x_{2}^{-\lambda-1}$ in Eq. (28) and take $\zeta\left(\epsilon / x_{2}\right) \simeq \zeta_{\max }$ (namely develop $\zeta$ in powers of $v=\epsilon / x_{2}$ and keep only the constant term) then

$$
\int_{\epsilon}^{1} d x_{2} g\left(x_{2}\right) \zeta\left(\frac{\epsilon}{x_{2}}\right) \simeq \zeta_{\max } \int_{\epsilon}^{1} d x_{2} x_{2}^{-\lambda-1} .
$$

Since $\epsilon \ll 1$, this integral is well approximated by $\zeta_{\max } \epsilon^{-\lambda} / \lambda$, for all $\lambda \neq 0$. Better approximations to the function $\zeta$ give similar results. For example, approximating the function $\zeta$ by two power laws, one above and another below the maximum, which is at about $x_{2}=5 \epsilon\left(\zeta=\zeta_{\max }\left(x_{2} / 5 \epsilon\right)^{2.1}\right.$ for $x_{2}$ between $\epsilon$ and $5 \epsilon$ and $\zeta=\zeta_{\max }\left(5 \epsilon / x_{2}\right)^{0.4}$ for $x_{2}$ between $5 \epsilon$ and 1), the integral in Eq.(32) becomes $\zeta_{\max }(5 \epsilon)^{-\lambda} /\left(0.9+1.7 \lambda-\lambda^{2}\right)$. Thus the essential dependence of $\epsilon^{-\lambda}$ is maintained. Recalling that $\epsilon=m_{c}^{2} /\left(2 m_{p} E_{c}\right)$, Eq. (19) is proportional to $E_{c}^{\lambda}$, and the same is true for Eq. (32), therefore

$$
\frac{d \phi_{c}}{d X}\left(E_{c}, X\right) \sim E_{c}^{-\gamma-1+\lambda}
$$

The charm production function $d \phi_{c}\left(E_{c}, X\right) / d X$, calculated numerically, is shown in Fig. 7a for a typical $X=$ $57.12 \mathrm{~g} / \mathrm{cm}^{2}(h=20 \mathrm{~km})$. We are using here the PDF MRS R1 with the three related values of $\lambda=0, \lambda(\mathrm{R} 1), 0.5$. We clearly see here that the slope at $E_{c} \gtrsim 10^{5} \mathrm{GeV}$ depends on the extrapolation of the gluon PDF at $x<10^{-5}$. This is one order of magnitude lower in energy than in Fig. 1 for the total cross section. This reflects the fact mentioned above that the characteristic charm energy is $E_{c}=\mathcal{O}(0.1 E)$. Fig. 7b shows that, as predicted analytically, the slopes (the negative of the spectral index in our notation) of the charm fluxes at production depend almost linearly on $\lambda$. In fact, in Fig. 7b, we can see that the logarithmic slopes of the $\lambda=0$ and $\lambda=0.5$ fluxes differ precisely by 0.5 , above 5 $10^{6} \mathrm{GeV}$ (namely, above the knee) to about $10^{9} \mathrm{GeV}$ (the maximum energy at which our fluxes are reliable, given that we take $10^{11} \mathrm{GeV}$ as the maximum incoming proton energy $E$ ). In fact, the slope of the $\lambda=0$ flux in that interval is about -3.1 to -3.2 , while that of the $\lambda=0.5$ flux is about -2.6 to -2.7 . Above the knee, the primary spectrum goes as $E^{\delta}$ with $\delta \simeq(-\gamma-1-0.1)=-3.1$, where we have also included the 0.1 contribution coming from the $E$-dependence of $\Lambda_{N}$ (see footnote in previous discussion), thus the charm spectrum, (in the energy range $10^{7} \mathrm{GeV} \lesssim E_{c} \lesssim 10^{9} \mathrm{GeV}$ ) goes approximately as $E_{c}^{\delta+\lambda}$ as expected from Eq. (33).

Using the definition of the leptonic fluxes in terms of the charm spectrum at production $d \phi_{c} / d X$, we can now find the dependence of the spectral index of muon and neutrino fluxes with $\lambda$. For example, the differential flux $\phi_{\mu}$ of muons with energy $E_{\mu}$ ( $\mu$ stands here for $\mu^{+}$or $\left.\mu^{-}\right)$is

$$
\phi_{\mu}\left(E_{\mu}\right)=2 \int_{X_{0}}^{\infty} d X \int_{E_{\mu}}^{\infty} d E_{c} \frac{d \phi_{c}\left(E_{c}, X\right)}{d X}\left[\frac{d N_{\mu}\left(c \rightarrow \mu ; E_{c}, E_{\mu}, X\right)}{d E_{\mu}}\right]
$$

( $\phi_{\mu}$ has, thus, units of $\left[1 / \mathrm{cm}^{2} \mathrm{~s} \mathrm{sr} \mathrm{GeV}\right]$ ). Here the factor of 2 accounts for the muons produced by $\bar{c}$ and the last square bracket is the number of muons of energy $E_{\mu}$ produced at sea level by the cascades, each cascade initiated by a $c$ quark of energy $E_{c}$ at a depth $X$.

Our results above indicate that we can write the atmospheric charm spectrum at production as (see Eq. (33)) $d \phi_{c}\left(E_{c}, X\right) / d X \simeq F(X) E_{c}^{-\gamma-1+\lambda}$ with $F(X)$ a function independent of energy. Replacing this form for $d \phi_{c}\left(E_{c}, X\right) / d X$ in Eq. (34) and multiplying and dividing by $E_{\mu}^{-\gamma-1+\lambda}$ we can write $\phi_{\mu}$ as

$$
\phi_{\mu}\left(E_{\mu}\right)=2 E_{\mu}^{-\gamma-1+\lambda} \int_{X_{0}}^{\infty} d X F(X) \int_{E_{\mu}}^{\infty} d E_{c}\left(\frac{E_{c}}{E_{\mu}}\right)^{-\gamma-1+\lambda}\left[\frac{d N_{\mu}\left(c \rightarrow \mu ; E_{c}, E_{\mu}, X\right)}{d E_{\mu}}\right] .
$$


We can argue that in so far as the values of the parent charm quark energy $E_{c}$ and the daughter lepton energy $E_{\mu}$ are not very different, the dependence of the integral on $\lambda$ (and on $\gamma$ ) should be mild. In this case, from Eq. (35), we find that the spectral index of the muon (and similarly of the neutrino) flux contains $\lambda$ as a term, i.e.

$$
\phi_{\mu}\left(E_{\mu}\right) \simeq f\left(E_{\mu}, \gamma, \lambda\right) E_{\mu}^{-\gamma-1+\lambda} \equiv E_{\mu}^{-b_{\mu}\left(E_{\mu}, \gamma, \lambda\right)+\lambda},
$$

where the dependence of the functions $f\left(E_{\mu}, \gamma, \lambda\right)$ and $b_{\mu}\left(E_{\mu}, \gamma, \lambda\right)$ on $\lambda$ and $\gamma$ should be mild. This justifies the results shown in Figs. 4 and 5, presented in Sect. IV], showing all the spectral indices obtained using all our PDF's.

Finally we examine the deviations from linearity of the relation between the spectral index $\alpha_{\ell}$ and the gluon PDF slope $\lambda$. In Fig. 8a we show directly the relation between $\lambda$ and $\alpha_{\ell}$, using the values coming from our simulation for the MRST case already presented in Fig. 4, but now plotting them for fixed energy $E_{\mu}$. We show two examples, for $E_{\mu}=1 \mathrm{PeV}, 10 \mathrm{PeV}$, where our points indicate a good agreement with the linear relation between $\alpha_{\ell}$ and $\lambda$ of Eq. (14).

The mild dependence on $\lambda$ of the functions $b_{\ell}(\lambda)=\alpha_{\ell}+\lambda$ can be seen in Fig. 8b, where we show the percentage difference $\left[b_{\ell}(\lambda)-b_{\ell}(0)\right] / b_{\ell}(0)$ for the different values of $\lambda=0-0.5$ with the MRST PDF. It is evident that, in the range where our theoretical arguments are applicable (for $E_{\mu} \gtrsim 10^{6} \mathrm{GeV}$ ) the $b_{\ell}(\lambda)$ functions differ only by $2-3 \%$ for different $\lambda$ values, namely they are almost independent of $\lambda$, given one particular PDF. This analysis confirms the validity of Eq. (14), which leads to the possibility of obtaining information on $\lambda$ at small parton fractions $x$ not reachable in experiments, through the measurement of the fluxes. We will study this possibility in more detail in a future paper [18].

\section{CONCLUSIONS}

The actual next-to-leading order perturbative QCD calculations of charm production cross sections, together with a full simulation of the atmospheric cascades, were used to obtain the vertical prompt fluxes of neutrinos and muons.

We have analyzed the dependence of the atmospheric fluxes on the extrapolation of the gluon PDF at very low $x$, which is related to the value of the parameter $\lambda$. This was done using four different sets of PDF's: MRS R1, MRS R2, CTEQ 4M and MRST, with variable $\lambda$ in the range $0-0.5$.

The charm production cross sections and the final lepton fluxes depend critically on $\lambda$ for leptonic energies $E_{l} \gtrsim$ $10^{5} \mathrm{GeV}$, which correspond to $x \lesssim 10^{-5} \mathrm{GeV}$. We found that the fluxes vary up to almost two orders of magnitude at the highest energy considered, $10^{9} \mathrm{GeV}$, for the different $\lambda$ 's in the allowed interval; on the contrary, for fixed $\lambda$, the results don't depend much on the choice of the PDF.

For the lowest values of $\lambda(\lambda \simeq 0-0.1)$ our fluxes are very close to those of TIG [6], confirming that the very low flux prediction is mostly due to a low value of $\lambda\left(\lambda_{\text {TIG }} \simeq 0.08\right)$. For higher values of $\lambda(\lambda \simeq 0.2-0.5)$ our results are in the bulk of previous predictions and, in particular, for $\lambda \simeq 0.3$ they are very close to a recent semi-analytical calculation [7] done with a similar value of $\lambda$.

We have also considered the dependence of the spectral index of the final fluxes on the parameters of the model. From both, computer simulations and analytical considerations, we find that the spectral index $\alpha_{\ell}$ of atmospheric leptonic fluxes depends linearly on $\lambda$ as in Eq. (14).

This suggests the possibility of obtaining bounds on $\lambda$ in "neutrino telescopes" for small values of $x$ not reachable in colliders, if the spectral index of leptonic atmospheric fluxes could be determined by these telescopes. We will investigate this possibility in detail in the future [18].

\section{ACKNOWLEDGMENTS}

The authors would like to thank the Aspen Center For Physics, where this work was initiated, for hospitality, and M. Mangano and P. Nason for the MNR program and helpful discussions. This research was supported in part by the US Department of Energy under grant DE-FG03-91ER40662 Task C. 
[1] T.K. Gaisser, "Cosmic Rays and Particle Physics" (Cambridge University Press, 1990).

[2] P. Nason, S. Dawson, and R.K. Ellis, Nucl. Phys. B303 (1988) 607.

P. Nason, S. Dawson, and R.K. Ellis, Nucl. Phys. B327 (1989) 49; Erratum B355 (1990) 260.

[3] M.L. Mangano, P. Nason, and G. Ridolfi, Nucl. Phys. B373 (1992) 295; Nucl. Phys. B405 (1993) 507.

[4] T. Sjöstrand, Computer Physics Commun. 82 (1994) 74.

[5] G. Gelmini, P. Gondolo, G. Varieschi, preprint hep-ph/9904457.

[6] M. Thunman, G. Ingelman, and P. Gondolo, Nucl. Phys. B (Proc. Suppl.) 43 (1995) 274.

P. Gondolo, G. Ingelman, and M. Thunman, Nucl. Phys. B (Proc. Suppl.) 48 (1996) 472.

M. Thunman, Ph.D. Thesis, Uppsala University, 1996.

M. Thunman, G. Ingelman, and P. Gondolo, Astroparticle Phys. 5 (1996) 309.

[7] L. Pasquali, M.H. Reno, and I. Sarcevic, preprint hep-ph/9806428.

[8] E769 Collaboration, G.A. Alves et al., Phys. Rev. Lett. 77 (1996) 2388.

[9] E769 Collaboration, G.A. Alves et al., Phys. Rev. Lett. 77 (1996) 2392.

[10] WA92 Collaboration, M.I. Adamovich et al., Nucl. Phys. B495 (1997) 3.

[11] S. Frixione, M.L. Mangano, P. Nason, and G. Ridolfi, preprint CERN-TH/97-16, hep-ph/9702287.

[12] A.D. Martin, R.G. Roberts and W.J. Stirling, Phys. Lett. 387 (1996) 419.

[13] H.L. Lai et al., Phys. Rev. D55 (1997) 1280.

[14] A.D. Martin, R.G. Roberts, W.J. Stirling and R.S. Thorne, preprint hep-ph/9803445.

[15] A.D. Martin, Acta Physica Pol. B25 (1994) 265.

J. Kwiecinski, Phys. Rev. D52 (1995) 1445.

[16] E.A. Kuraev, L.N. Lipatov and V.S. Fadin, Sov. Phys. JETP 45 (1977) 199.

Ya. Ya. Balitsky, L.N. Lipatov, Sov. J. Nucl. Phys. 28 (1978) 822.

[17] Review of Particle Physics, Phys. Rev. D54 (1996) 1.

[18] G. Gelmini, P. Gondolo, G. Varieschi, in preparation. 


\section{FIGURE CAPTIONS}

Fig. 1 Total cross section for charm production $\sigma_{c \bar{c}}$, up to NLO, for our different PDF's and $\lambda$ values, compared to that used by TIG [6]. Top panel: MRS R1-R2 and CTEQ 4M; bottom panel: MRST (cross sections increase with $\lambda$ ).

Fig. 2 Prompt muons: $E^{3}$-weighted vertical fluxes at NLO, compared to the TIG [6] conventional and prompt fluxes (dotted lines). We show results using the four PDF's MRS R1, MRS R2, CTEQ 4M and MRST.

Fig. 3 Prompt muon-neutrinos: $E^{3}$-weighted vertical fluxes at NLO, compared to the TIG [6] conventional and prompt fluxes (dotted lines). We show results using the four PDF's MRS R1, MRS R2, CTEQ 4M and MRST.

Fig. 4 Prompt muons: spectral index of the NLO vertical fluxes for the four PDF's MRS R1, MRS R2, CTEQ 4M and MRST.

Fig. 5 Dependence of prompt fluxes and their spectral index on the PDF at fixed $\lambda$ : left side $\lambda=0$, right side $\lambda=0.5$.

Fig. 6 (a) The function $\zeta_{g g}(v)$ at the Born level for $\gamma=0,1.7$ (below the knee) and $\gamma=2$ (above the knee). (b) Flux-weighted charm production spectra $x_{E}^{\gamma} \frac{1}{\sigma} \frac{d \sigma}{d x_{E}}$ at several beam energies (using MRS R1, $\lambda(\mathrm{R} 1)$ ).

Fig. 7 (a) NLO charm production function $E_{c}^{3} d \phi_{c}\left(E_{c}, X\right) / d X$ (PDF MRS R1); (b) its spectral index $-\partial \ln \left[\partial \phi_{c}\left(E_{c}, X\right) / \partial X\right] / \partial \ln E_{c}$. These results are for a height $h=20 \mathrm{~km}$, corresponding to a vertical depth $X=57.12 \mathrm{~g} / \mathrm{cm}^{2}$ (similar results are obtained for other heights).

Fig. 8 (a) Relation between the slope $\lambda$ of the gluon PDF and the muon spectral index $\alpha_{\mu}$ at fixed muon energy. (b) Non-linearities in this relation. Here $b_{\ell}(\lambda)=\alpha_{\ell}(\lambda)+\lambda$ and we use the MRST PDF. 
Gelmini, Gondolo, Varieschi 1999
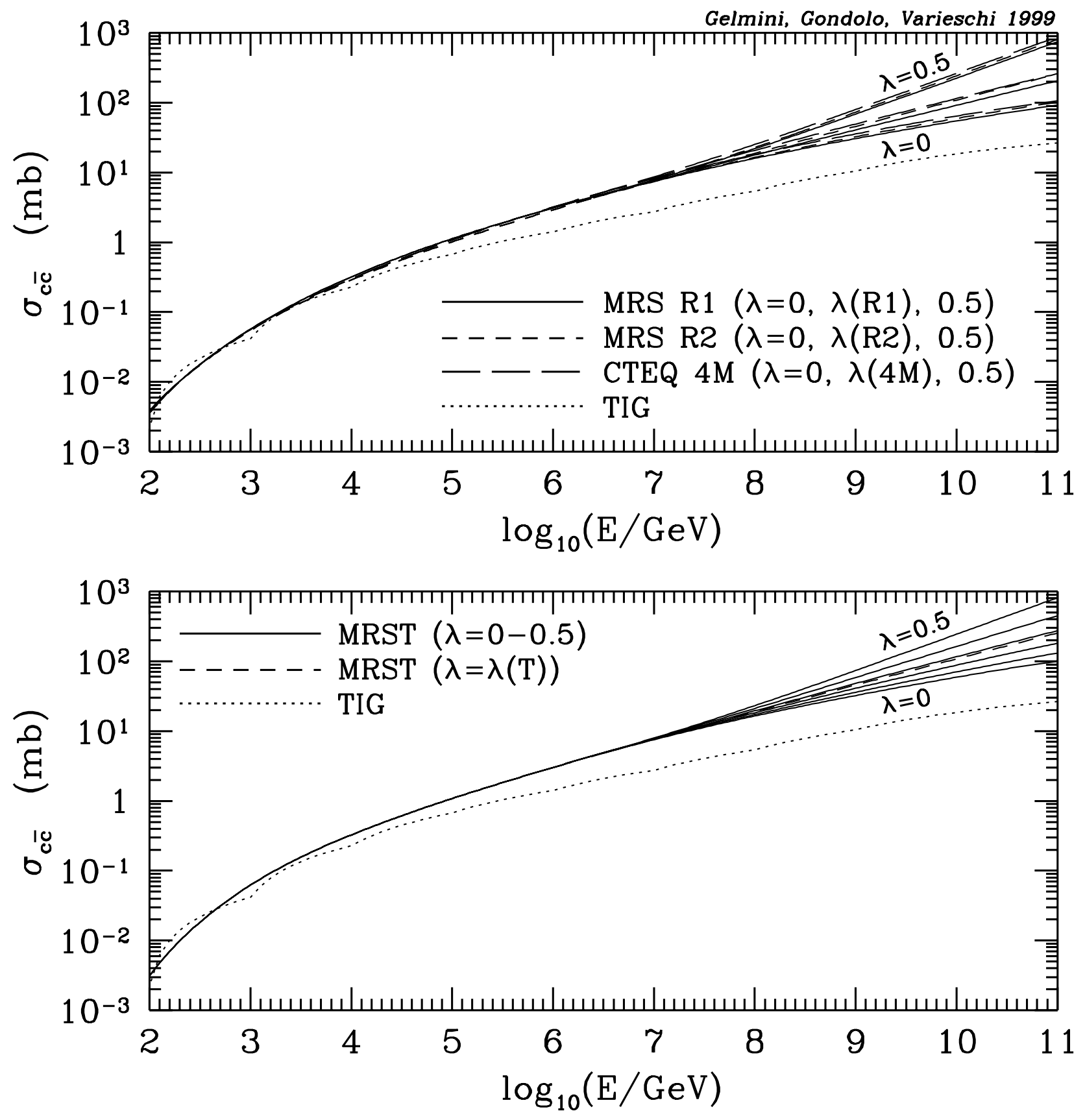

FIG. 1. 


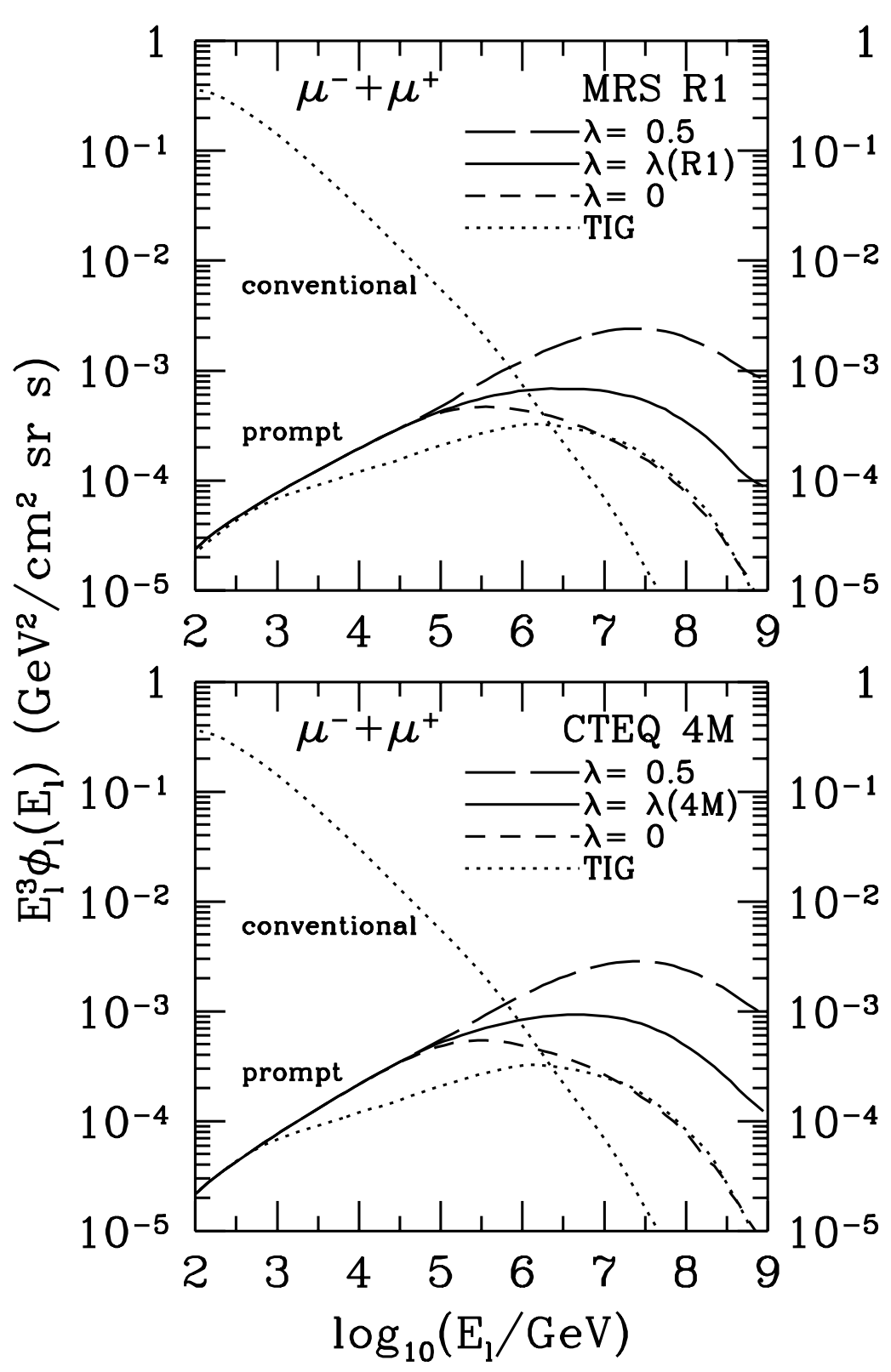

Gelmini, Gondolo, Varieschi 1999
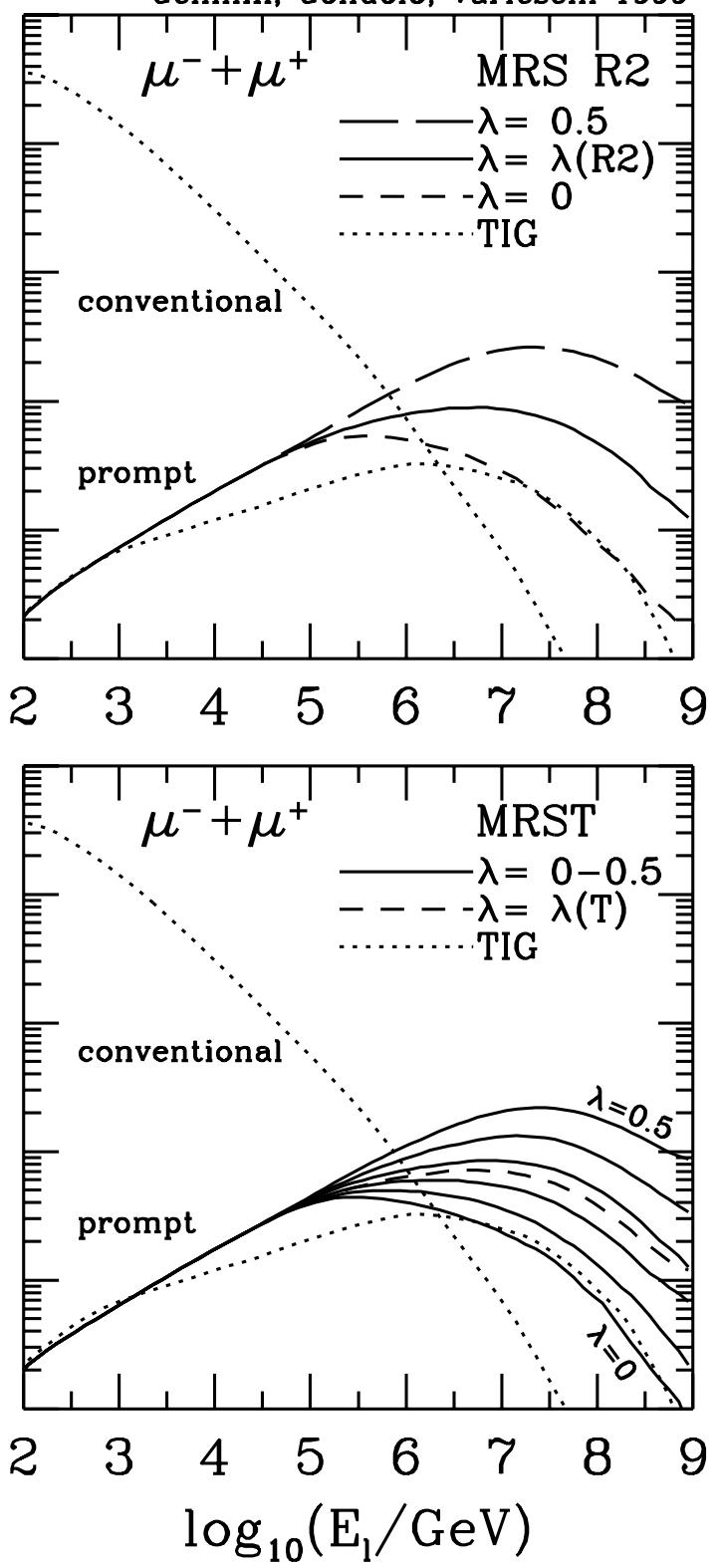

FIG. 2. 

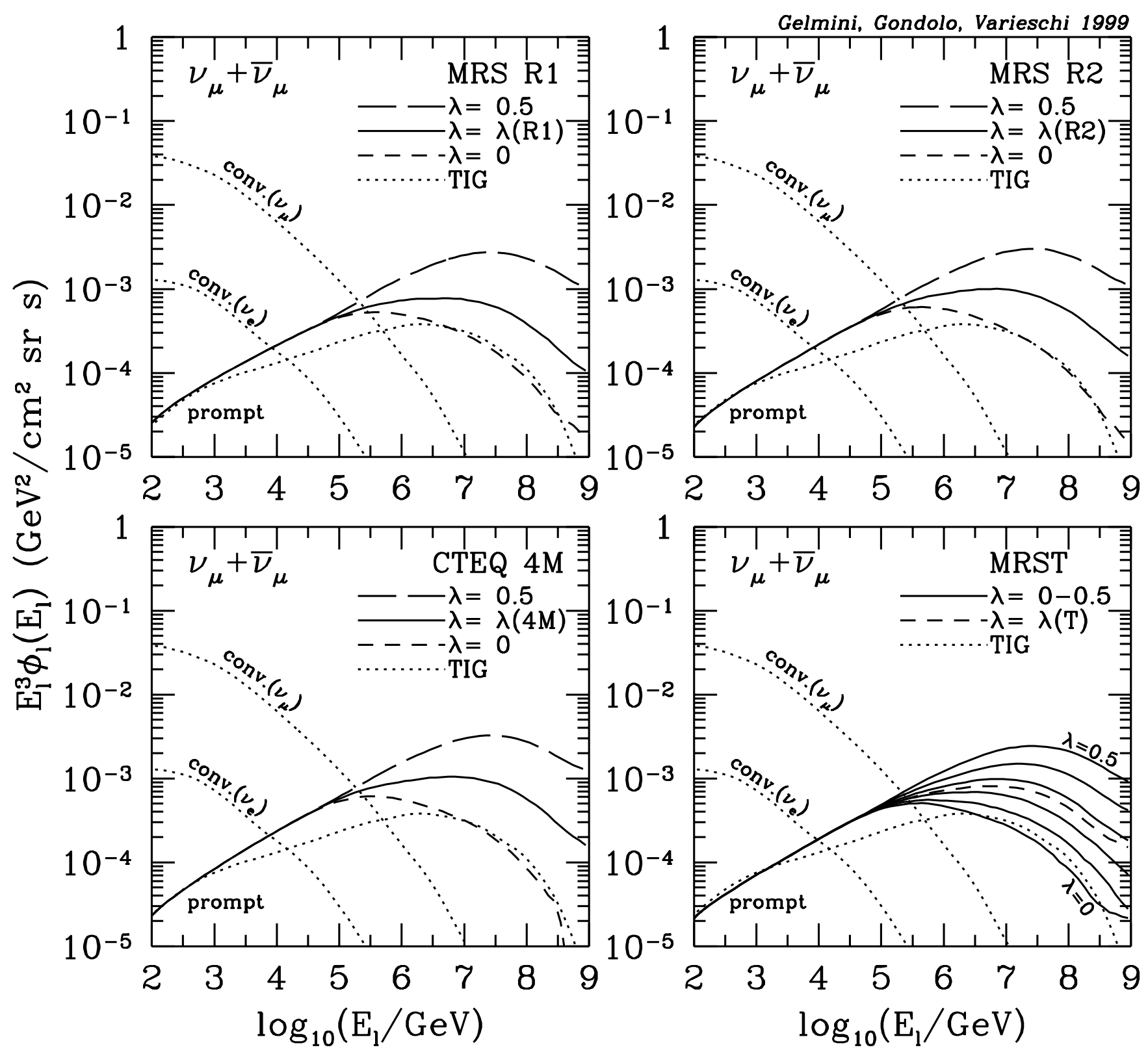

FIG. 3. 

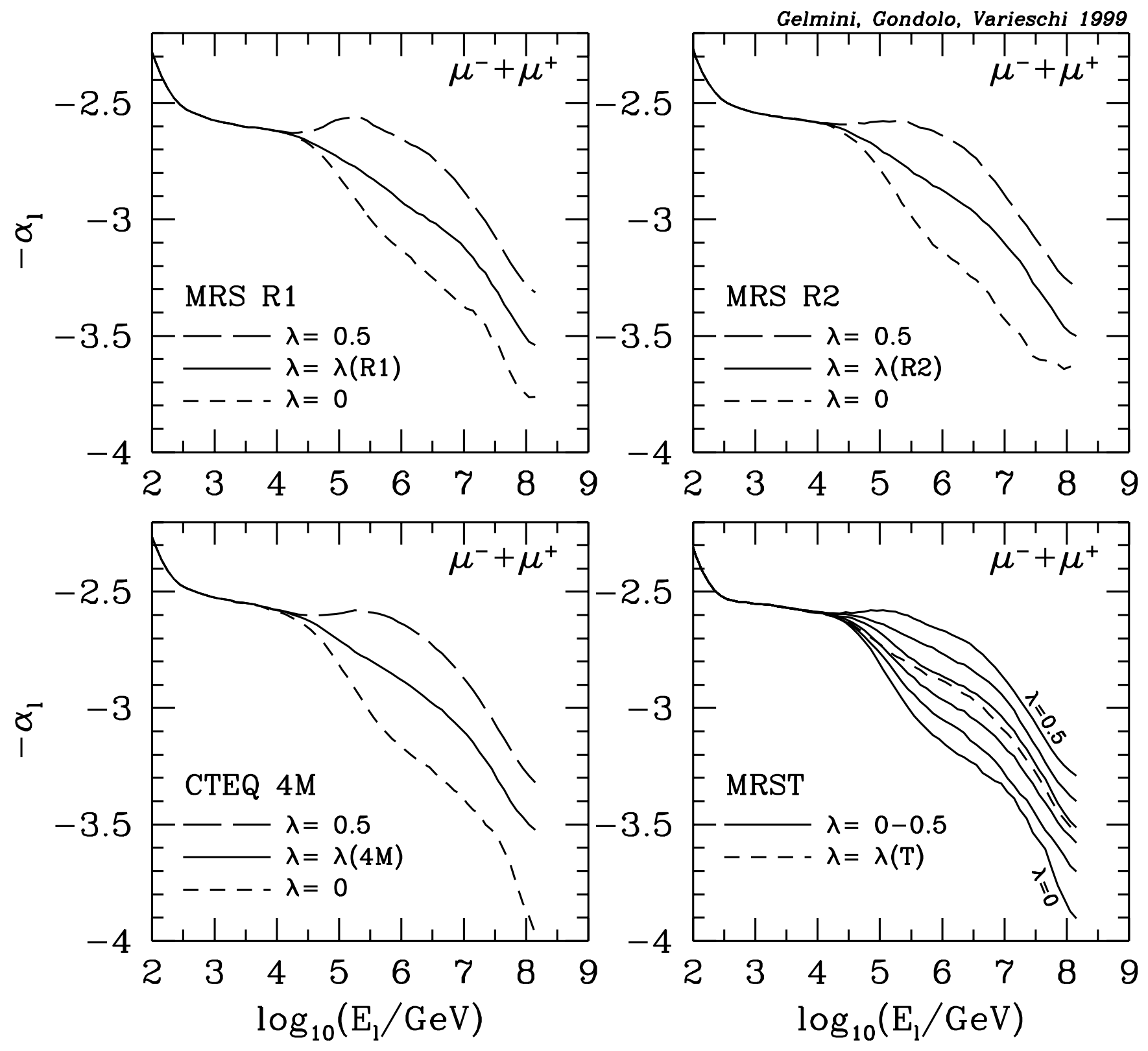

FIG. 4. 

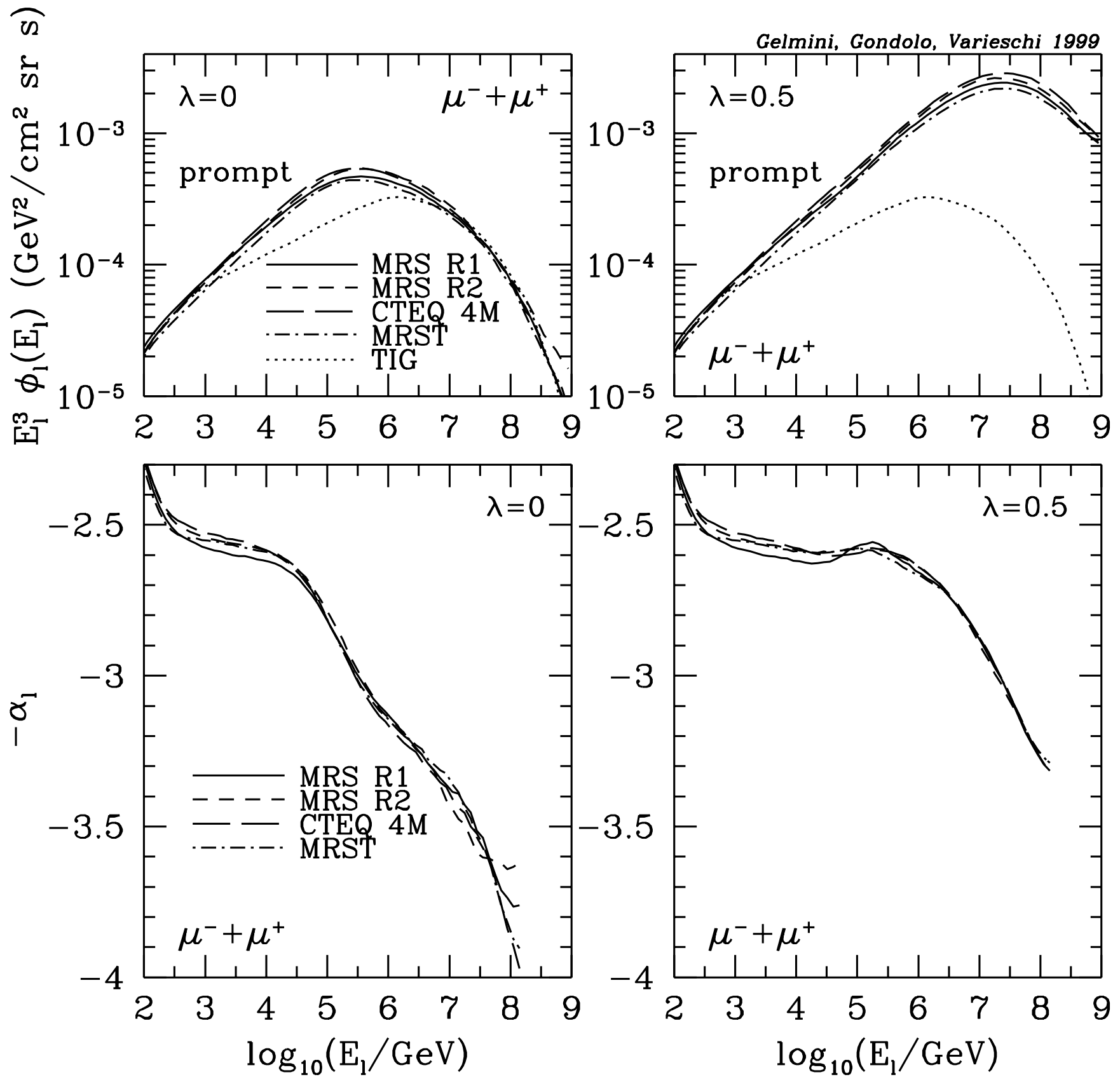

FIG. 5. 

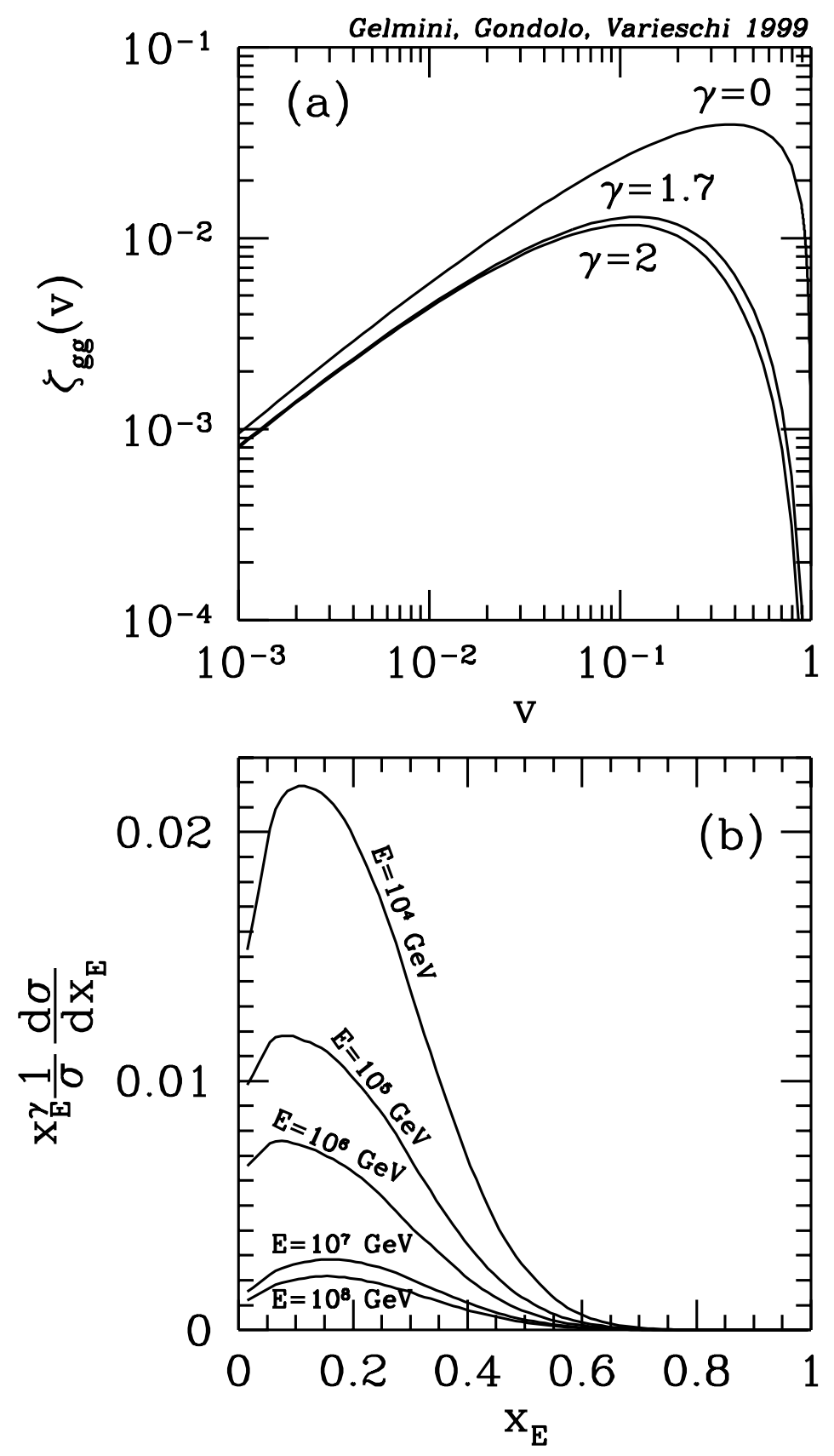

FIG. 6. 

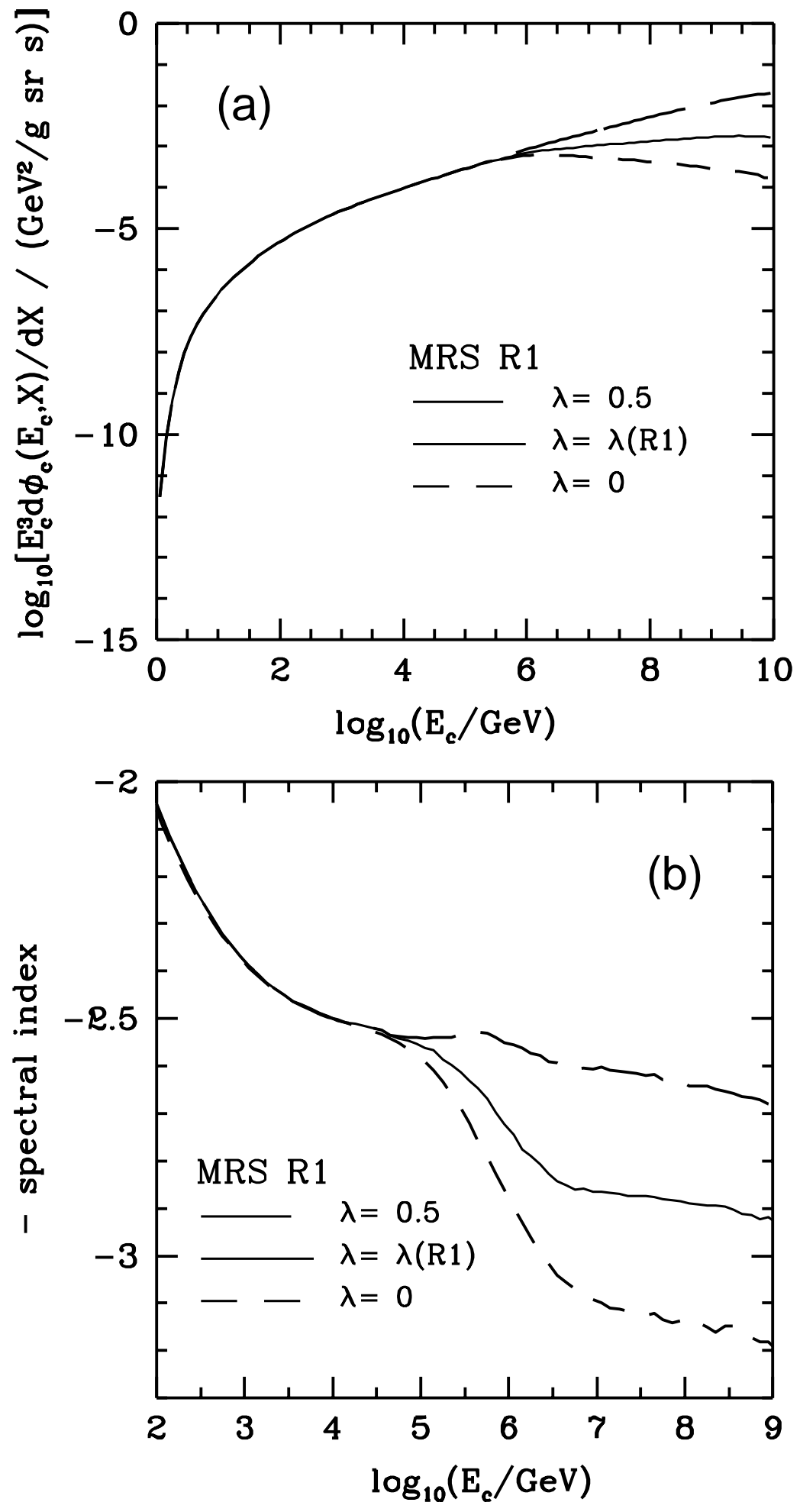

FIG. 7. 

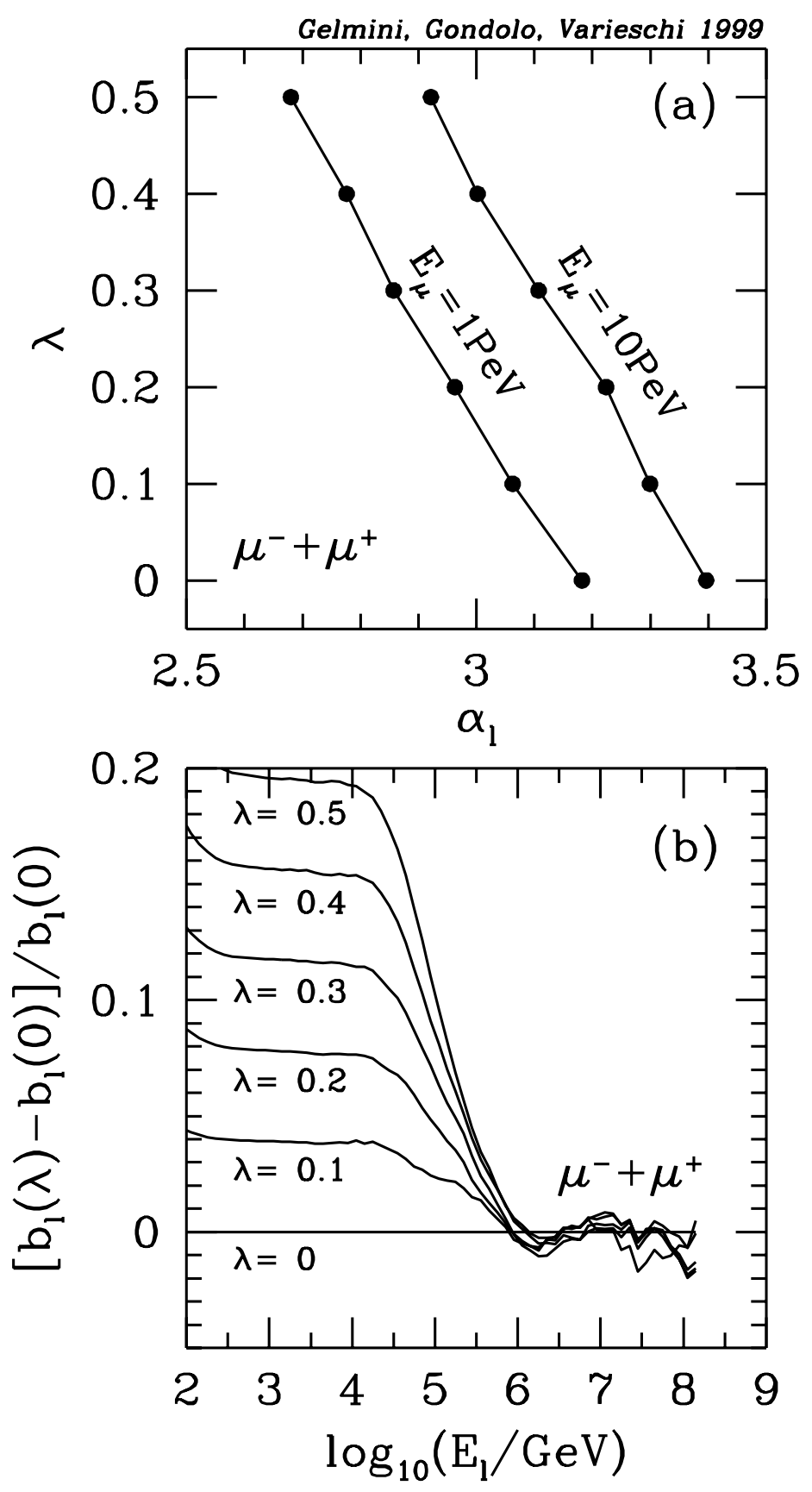

FIG. 8. 